\title{
Matrix metalloproteinase in the cardiovascular remodeling of hypertension: current insights and therapeutic potential
}

This article was published in the following Dove Press journal:

Metalloproteinases In Medicine

Juliana M Parente
Michele M Castro

Department of Pharmacology, Ribeirao Preto Medical School, University of Sao Paulo, Ribeirão Preto, SP, Brazil
Correspondence: Michele M Castro Department of Pharmacology, Ribeirao Preto Medical School, University of Sao Paulo, Av. Bandeirantes 3900, 14049-900 Ribeirão Preto, SP, Brazil

$\mathrm{Tel}+551633153038$

Fax +551633150220

Email castro@fmrp.usp.br

\begin{abstract}
Hypertension induces maladaptive vascular and cardiac remodeling, which are related to rearrangement of the extracellular matrix (ECM) and cell hypertrophy and migration. Matrix metalloproteinases (MMPs) are zinc-dependent proteases involved in tissue remodeling mainly by the proteolysis of ECM components. Increased MMP-2 activity is also involved in the proteolysis of important intracellular targets in cardiomyocytes and vascular smooth muscle cells (VSMC). Troponin I and calponin-1 are some of the targets of MMP-2 in cardiomyocytes and VSMC, respectively, that when degraded contribute to contractile dysfunction, cell hypertrophy or migration. MMP-2 may be activated by S-glutathiolation in vitro by peroxynitrite which frees the pro-peptide domain from the catalytic site and generates an active, $72 \mathrm{kDa}$ MMP-2. Since hypertension is significant related to oxidative stress, and approximately half of newly formed MMP-2 is held inside the cell, increased peroxynitrite production may lead to the intracellular activation of MMP-2. MMP inhibitors may be a significant new opportunity to be used as adjuvants to treat hypertension as they substantially decrease maladaptive cardiovascular remodeling and then prevent the development of many other associated diseases. Antioxidants and antihypertensive drugs also contribute to decrease MMP activity and hypertrophic remodeling in hypertension. New pharmacological tools are needed to specifically decrease intracellular MMP-2 activity and thus help reduce cell migration and hypertrophy in hypertension.
\end{abstract}

Keywords: hypertension, matrix metalloproteinases, cardiovascular remodeling, matrix metalloproteinase inhibitors, antioxidants

\section{Introduction}

Matrix metalloproteinases (MMPs) are zinc-dependent proteases that are involved in the proteolysis of several extracellular matrix (ECM) components in cardiomyocytes and vascular smooth muscle cells (VSMC), which may result in cell hypertrophy, proliferation and/or migration. These effects contribute to chronic cardiovascular remodeling and dysfunction that are associated with the maladaptive consequences of hypertension. ${ }^{1-9}$ However, the roles of MMPs are not only restricted to ECM proteolysis. Increased MMP-2 activity was also associated with intracellular effects in cardiomyocytes and VSMC where it degrades proteins in the contractile machinery such as troponin I, titin and calponin-1, thus contributing to maladaptive remodeling of the heart and vasculature. ${ }^{1,2,10-12}$ As increased MMP activity contributes to structural and functional changes in the cardiovascular system in hypertension, it is promising to consider MMPs as pharmacological targets to treat or prevent many cardiovascular complications. Increased activity of MMPs is also relevant to study structural and functional alterations in the 
kidney and brain in hypertension, however we will focus here on their actions within the cardiovascular system.

\section{MMPs: definition of structure and regulation}

MMPs are a group of 28 endopeptidases that were first described by Gross and Lapiere in $1962,{ }^{13}$ who observed their collagen-degrading activity during the morphogenesis of tadpoles. Today, MMPs are known as important proteases involved in proteolysis of ECM in tissue remodeling in many pathophysiological situations. ${ }^{14,15}$ MMPs were originally classified according to their ECM substrates, primary structure and cellular location as collagenases (MMP-1, MMP-8 and MMP-13), stromelysins (MMP-3, MMP-10, MMP-11 and MMP-12), matrilysins (MMP-7 and MMP-26), membrane type MMPs (MT1-MMP to MT6-MMP) and gelatinases (MMP-2 and MMP-9). ${ }^{16-18}$ Although they are classified by their substrate specificity, many MMPs also share proteolysis of the same targets such as collagen and elastin. ${ }^{19}$ However, it should be noted that this system of classification precluded the discovering and therefore the actions and substrates of intracellular MMPs.

MMPs have important similarities in their structure: a pre-peptide domain (or signal sequence) followed by a propeptide region linked to the catalytic site, and a hemopexin domain. The pro-peptide domain protects the catalytic site of MMPs against activation or autolysis and maintains the enzyme in a latent form. Next to the catalytic site, the hemopexin domain is the region where tissue inhibitors of metalloproteinases (TIMPs) bind to MMPs to inhibit their activity. ${ }^{16,17,20,21}$ Among all MMPs, MMP-2 has been studied as the principal protease involved in hypertension-induced cardiovascular remodeling as it exerts its effects on both the ECM and within the cardiomyocytes and VSMC. These effects contribute to cell hypertrophy, proliferation and/or migration. ${ }^{10,22} \mathrm{MMP}-2$ is found in all cells, including VSMC, cardiomyocytes, endothelial cells and platelets, although it is also regulated and activated by oxidative stress.

The N-terminal pre-peptide domain of MMP-2 serves as a signal sequence for its secretion to the extracellular environment after being synthetized..$^{17,19,20,23}$ However, it was shown that there are also: (1) an N-terminal splice variant of MMP-2 (MMP-2 ${ }_{\text {NTT-50 }}$ ) that lacks the first 50 amino acids of the signal sequence and thereby retains it within the cytosol of cardiomyocytes and other cells, ${ }^{23,24}$ (2) a signal sequence of canonical MMP-2 that is inefficient to direct itself to the endoplasmic reticulum and the secretory pathway, thus resulting in approximately half of MMP-2 that resides in the cytosol; ${ }^{24}$ and (3) another N-terminal truncated isoform of MMP-2 (MMP-2 ${ }_{\text {NTT-76 }}$ ) which is expressed in response to oxidative stress. ${ }^{23}$ As there are at least three isoforms of MMP-2 inside cells, the probability that intracellular activation of MMP-2 occurs by S-glutathiolation or phosphorylation is significant, and this opens new fields of investigation.

When canonical $72 \mathrm{kDa}$ MMP-2 is secreted from cells, it is activated by its complex with membrane type 1 (MT1)MMP and TIMP-2 in plasma membrane, through proteolytic removal of the pro-peptide, which renders it as an active form of $64 \mathrm{kDa}$ MMP-2. ${ }^{17,20,23}$ Figure 1 shows MT1-MMP in the cell membrane of VSMC and $64 \mathrm{kDa}$ MMP-2 released from cells to proteolyze ECM products. Other extracellular proteases, such as serine proteases, can also activate MMP-2. The proteolytic activation of MMP-2 usually requires the binding of TIMP-2 to MT1-MMP in the plasma membrane, which allows MMP-2 to anchor to this complex. Then another molecule of MT1-MMP cleaves the pro-peptide domain of MMP-2, thus activating it. There are several critical reviews which discuss this process..$^{20,21,23}$ The catalytic domain of MMP-2 has a conserved zinc-binding motif and three fibronectinlike domains, which give MMP-2 the specificity to degrade denatured collagen and type IV collagen, for example. By degrading these ECM components, MMP-2 contributes to migration and proliferation of VSMC, and then it results in the maladaptive vascular remodeling of hypertension. ${ }^{22}$ The catalytic site of MMP-2 is followed by a C-terminal region that contains a hemopexin-like domain. These structures are connected by a hinge region. The hemopexin domain favors the binding of MMP-2 to TIMP-2, for example, thus contributing to the inhibition of MMP-2 activity. ${ }^{17,19,20,23}$

MMPs are regulated by gene transcription, zymogen activation, ${ }^{21}$ posttranslational modifications (glutathiolation, nitrosylation and phosphorylation $)^{26-28}$ and binding to TIMPs that inhibit their activity. ${ }^{17}$ Four unique TIMPs were identified and although they exhibit a broad MMP inhibitory profile, they share some specificities. TIMP-1 preferentially inhibits MMP9, while TIMP-2 inhibits MMP-2. TIMP-3 is an important marker of cardiac hypertrophy and inhibits almost all MMPs in the ECM, and TIMP-4 inhibits MT1-MMP and MMP-2 and is found in both the ECM and inside of cardiomyocytes where it colocalizes with MMP-2. ${ }^{17,29,30}$ The N-terminal region of TIMPs binds to the catalytic domain of MMPs to inhibit their activity and the C-terminal of TIMPs binds to the hemopexin domain of MMPs, thus stabilizing the inhibitory complex. ${ }^{17,29}$

S-glutathiolation ${ }^{26}$ and phosphorylation ${ }^{27}$ are significant posttranslational alterations that regulate the activity of MMP-2 inside the cell. Low micromolar concentrations of 




Figure I Increased MMP-2 activity and the proteolysis of ECM and non-ECM components in the VSMC. Increased MMP-2 activity contributes to hypertension-induced maladaptive vascular remodeling as it degrades many ECM and non-ECM components in the VSMC. MMP-2 is activated by MTI-MMP in the ECM, where it degrades collagen and elastin. The cleaved products of collagen interact with the integrin receptors in the VSMC and activate the focal adhesion kinase signaling, which stimulates migration and proliferation. In the ECM, MMP-2 also processes the latent TGF- $\beta$ to facilitate VSMC migration and the $\beta_{2}$ adrenergic and insulin receptors, which leads to vasoconstriction. MMP-2 may also cleave cadherin between VSMC. The activation of MMP-2 by oxidative stress may contribute to proteolysis of calponin-I in the machinery of VSMC, thus resulting in migration or proliferation. In the endothelial cells, MMPs may cleave the VEGFR-2 and P-selectin, and then participate in many inflammatory processes in the vasculature during hypertension. HSP90 and eNOS may be potential targets of MMP-2 in endothelial cells, thus contributing to hypertension-induced vascular endothelial dysfunction. Finally, MMP-2 also cleaves the IL-I $\beta$ that increases reactive oxygen species and increases proliferation in VSMC. The use of antioxidants and some antihypertensive drugs also reduces increased MMP-2 activity by decreasing oxidative stress in the VSMC.

Abbreviations: BETA 2, $\beta_{2}$ adrenergic receptor; CCB, calcium channel blockers; ECM, extracellular matrix; eNOS, endothelial nitric oxide synthase; EGFR, epidermal growth factor receptor; HSP90, heat shock protein 90; IL-I $\beta$, interleukin-I beta; MMP, metalloproteinase; MTI-MMP, membrane type-I metalloproteinase; ONOO-, peroxynitrite; TGF- $\beta$, transforming growth factor- $\beta$; VEGFR-2, vascular endothelial growth factor receptor; VSMC, vascular smooth muscle cells.

peroxynitrite, in the presence of cellular glutathione, disrupt the coordination bond of cysteinyl thiol in the MMP-2 pro-peptide domain with zinc in the catalytic site. This process changes the conformation of the pro-peptide domain of MMP-2, thus forming the glutathione disulfide S-oxide, $\mathrm{GS}(\mathrm{O}) \mathrm{SR}$, which frees the catalytic domain of MMP-2 and allows the proteolysis of many substrates. ${ }^{26}$ In aortas of lipopolysaccharide (LPS)-injected rats, glutathione was co-immunoprecipitated together with the $72 \mathrm{kDa}$ MMP-2, which may suggest that MMP-2 is potentially activated by S-glutathiolation. This mechanism was associated with the loss of calponin-1 in aortas of LPS-injected rats, which was prevented by an MMP inhibitor. These possibilities of activating MMP-2 inside the cells open a new field of investiga- tion to inhibit the intracellular forms of MMP-2 in order to prevent proteolysis of the intracellular matrix that occurs in cardiovascular pathologies associated with oxidative stress.

\section{MMP inhibitors}

Several synthetic inhibitors inhibit MMP activity. Golub et $\mathrm{a}^{31,32}$ studied gingival inflammation and periodontitis in rats and discovered that some tetracyclines had an MMP inhibitory activity independent of their antibacterial actions. Since then, several studies have shown that doxycycline is an MMP inhibitor already at a subantimicrobial plasma concentration. Chemically modified tetracyclines were synthetized which were devoid of antibiotic effect but were able to inhibit MMP activity. ${ }^{33}$ The MMP inhibitors doxycycline, 
ONO-4817 (hydroxamate), BAY12-9566 (carboxylate) and the sulfonamide-based ARP-100 are all zinc chelators, which through this property inhibit MMP activity. The hydroxamates are generally more potent than carboxylates in inhibiting MMPs; however, at acidic $\mathrm{pH}$, characteristic of the inflammatory environment, the protonated carboxylates may become more potent to inhibit MMPs. ${ }^{34}$ ARP-100 is considered to be an MMP-2-preferring inhibitor as it has biphenyl sulfonamide groups in its structure, which are important for inhibiting MMP-2 $(\mathrm{Ki}=12 \mathrm{nM}) .{ }^{35}$ On the other hand, ONO-4817, BAY12-9566 and doxycycline are more broad-spectrum MMP inhibitors with different potencies to inhibit MMP-2 $\left(\mathrm{Ki}=0.73 \mathrm{nM},{ }^{36} \mathrm{Ki}=11 \mathrm{nM},{ }^{37}\right.$ and $\mathrm{Ki}=30-50$ $\mu \mathrm{M},{ }^{38}$ respectively). Since MMPs, especially MMP-2, are involved in hypertension-induced cardiovascular remodeling, their inhibition may be a good strategy to ameliorate the chronic alterations of hypertension..$^{39-41}$

\section{Inhibition of ECM proteolysis by MMPs ameliorates hypertension- induced maladaptive remodeling}

To facilitate remodeling of blood vessels and heart chambers, ECM components need to be cleaved to facilitate cell proliferation, migration and/or hypertrophy. For vessels, there are three major types of remodeling: hypotrophic, hypertrophic and eutrophic. To adapt the vessels to the increased wall stress of hypertension, hypertrophic remodeling occurs and is represented by thickening of the arterial media and increased proliferation of VSMC, which generally lead to increased media-to-lumen ratio and cross-sectional area (CSA). This hypertrophic remodeling is also associated with the resynthesis of many ECM components (such as collagen) in the conductance arteries, thus also contributing to hypertensioninduced rigidity. ${ }^{15}$ Eutrophic remodeling is another common situation in hypertension that mostly occurs in resistance arteries. The VSMC rearrange themselves into the medial layer after migration to reduce the lumen size in order to adapt the resistance arteries to wall stress. This process generally occurs without any change in the CSA.${ }^{42}$ On the other hand, hypotrophic vascular remodeling is mainly characterized by a significant decrease in the CSA and/or media-to-lumen ratio of resistance arteries, and generally occurs when blood flow is reduced. Increased apoptosis and/or atrophy of some layers of the vascular wall might be involved in this type of remodeling. ${ }^{43,44}$

To contribute to chronic vascular remodeling of hypertension, MMP-2 can degrade collagen type IV, a component of the basement membrane of VSMC, which facilitates migration and proliferation. ${ }^{14}$ Furthermore, collagen type IV cleavage by MMP-2 contributes to an increase in the synthesis of collagen type I, elastin and tenascin in VSMC, which contributes to vascular wall thickening. In fact, when collagen is degraded, its cleavage products can bind to different integrins in the VSMC and then trigger migration and proliferation in addition to the synthesis of new ECM components. Figure 1 shows a representation of degradation products of collagen binding to the integrin receptor in VSMC. Results from our group showed that increased MMP-2 activity was associated with chronic, maladaptive, vascular remodeling and contractile dysfunction in aortas of rats subjected to the two kidney-one clip (2K-1C) model of hypertension. These changes were associated with increased deposition of collagen and elastin in aortas as a consequence of increased hypertrophy and hyperplasia in VSMC., ${ }^{3,5-7}$ It is important to note that once elastin is cleaved and then resynthesized, it loses its original elastic capacity, which thus impairs arterial plasticity. Treatment with doxycycline ameliorated both arterial endothelial dysfunction and hypertrophic remodeling in $2 \mathrm{~K}-1 \mathrm{C}$ rats. ${ }^{3}$ Doxycycline also reduced vasoconstriction and eutrophic remodeling in mesenteric arteries of one-week $2 \mathrm{~K}-1 \mathrm{C}$ or L-NAME (N(G)-nitro-Larginine methyl ester) hypertensive rats, respectively. ${ }^{2,45}$ Increased MMP activity also contributes to the proteolysis of aortic elastin and increased collagen deposition observed in 24-month-old rats. Treatment with PD-166739, an MMP inhibitor, reduced age-induced hypertension and decreased MMP activity, elastin proteolysis and collagen deposition in the arteries. ${ }^{46}$ Moreover, increased transmural pressure in porcine carotid arteries resulted in increased MMP-2 and MMP-9 activities and decreased elastin levels. This may result in hypertensive vascular remodeling. ${ }^{47}$ Figure 1 shows $64 \mathrm{kDa}$ MMP-2 associated with collagen and elastin in the ECM of VSMC to cleave them.

In the heart, when systolic blood pressure increases, the left ventricles undergo hypertrophic, concentric remodeling to adapt themselves to the increased wall tension. Therefore, cardiac concentric remodeling is characterized by a significant hypertrophy of the left ventricle wall, hypertrophic cardiomyocytes and reduced chamber size. This hypertrophy may contribute to hypertension-induced increase in cardiac contractility. This remodeling may shift to hypertrophic, eccentric remodeling, which involves a significant dilation of the left ventricle chamber, increased deposition of collagen in the ventricle wall and a significant reduction in the contractility, which may lead to heart failure..$^{48}$ As MMP-2 is known to cleave ECM and also intracellular targets in the 
contractile machinery of cardiomyocytes, it may generate these structural alterations in hypertension. ${ }^{15,49,50}$ In fact, increased MMP-2 activity was associated with hypertrophic, concentric remodeling and increased cardiac contractility in $2 \mathrm{~K}-1 \mathrm{C}$ rats, and doxycycline ameliorated such chronic alterations. ${ }^{8}$ Furthermore, 8-month-old transgenic mice which express active MMP-2 only in the heart showed increased MMP-2 activity, collagen deposition, decreased left ventricle ejection fraction and hypertrophic, eccentric remodeling. Therefore, increased MMP-2 activity per se is important to induce cardiac remodeling and failure. ${ }^{51}$

\section{MMP inhibition reduces proteolysis of non-ECM targets in hypertension}

Another extracellular target of MMP-2 is transforming growth factor (TGF)- $\beta$. TGF- $\beta$ is a proinflammatory cytokine involved in collagen and fibronectin synthesis in VSMC and cardiomyocytes by triggering the signaling pathway of small mothers against decapentaplegic protein (SMADs). TGF- $\beta$ also regulates proliferation, apoptosis, differentiation and migration of VSMC. MMP- 2 activates latent TGF- $\beta$ by cleavage of the latency-associated peptide in its structure, and this effect contributes to cause the cardiovascular hypertrophic remodeling of hypertension. ${ }^{52,53}$ Figure 1 also shows MMP-2 contributing to the activation of TGF- $\beta$ in the membrane of VSMC. Thus, MMP inhibition ameliorated these changes by reducing proliferation of VSMC and collagen deposition in the ECM of the heart and vasculature. ${ }^{53-55}$

MMP-2 may also cleave cadherin, molecules that help to connect VSMC attached to each other (Figure 1). Therefore, disruption of cadherin may led to migration of VSMC and then vessel remodeling. ${ }^{23}$ In deoxycorticosterone acetate-salt hypertensive rats, increased MMP-2 activity contributed to reduced levels of E-cadherin and increased fibrosis in the proximal tubule of the kidney. Treatment with MMP inhibitor decreased MMP-2 activity and restored the levels of E-cadherin in the kidneys, thus ameliorating fibrosis. ${ }^{56}$ Moreover, N-cadherin proteolysis by MMPs led to proliferation of VSMC via $\beta$-catenin signaling in vitro. ${ }^{57}$

MMPs also degrade other non-ECM targets which generate vasoconstrictors. MMP-2 cleaves vasoactive peptide precursors such as big endothelin-1, which generates a potent vasoconstrictor peptide (ET-1 [to 32]). ${ }^{58}$ In fact, an increased vasoconstrictor response to big endothelin-1 in the mesenteric arteries of rats submitted to reduced uterine artery perfusion pressure was associated with increased MMP-2 activity as it generated the potent vasoconstrictor, endothelin-1. ${ }^{59} \mathrm{MMP}-2$ cleaves calcitonin gene-related peptide to reduce its vasodila- tory capacity, ${ }^{60}$ and adrenomedullin to form vasoconstrictor products. ${ }^{61}$ In fact, in lead-induced hypertension in rats, doxycycline prevented reduction in circulating adrenomedullin and the increase in systolic blood pressure, thus suggesting that inhibiting MMP activity preserves adrenomedullin and its important vasodilatory effects. ${ }^{62}$

MMP-2 also contributes to the proteolysis of heat shock protein (HSP90), a cofactor of endothelial nitric oxide synthase in the fructose-fed hypertensive rats (Figure 1). Doxycycline reduced MMP-2 activity and restored endothelial nitric oxide synthase and HSP90 protein levels in the rat mesenteric arteries, which improved the endothelial function. ${ }^{63} \mathrm{MMP}-2$ also cleaves receptors in the VSMC. Figure 1 shows MMP-2 closely associated to the $\beta_{2}$ adrenergic receptor and insulin receptor in the VSMC membrane. In fact, in a study of blood vessels from spontaneously hypertensive rats (SHR), MMPs contributed to the proteolysis of the extracellular domain of $\beta_{2}$ receptor, which resulted in vasoconstriction. These effects were prevented by using doxycycline. ${ }^{64}$ On the other hand, proteolytic cleavage of the insulin receptor-binding domain by MMPs was associated with oxidative stress in the SHR mesenteric arteries. Inhibition of MMP activity with doxycycline reduced systolic blood pressure and oxidative stress in addition to reducing glucose transport into the cells and normalizing blood glucose and glycol-hemoglobin levels in SHR. ${ }^{65}$ Another MMP substrate in the vasculature of hypertensive rats is vascular endothelial growth factor receptor (VEGFR-2). MMP inhibition with doxycycline attenuated VEGFR-2 cleavage and prevented capillary rarefaction in SHR. ${ }^{66}$ MMP-2 knockout mice prevented angiotensin IIinduced endothelial dysfunction, vascular remodeling, oxidative stress and inflammation. ${ }^{67}$ These effects were related to reduced activation of epidermal growth factor receptor and the signaling pathways such as extracellular signal-regulated kinases (ERK-1/2) and mitogen-activated protein kinases in VSMC. ${ }^{67}$

\section{MMPs may degrade inflammatory targets and contribute to hypertension-induced maladaptive cardiovascular effects}

Hypertension is an inflammatory condition and the increased levels of MMPs in the arteries contribute to activate many cytokines. On the other hand, increased cytokines and oxidative stress also contribute to increase MMP activity and their proteolytic actions in the vasculature. While MMP-2 is constitutively expressed in the heart and vasculature, MMP-9 is an inducible protease and its presence in tissues is associated 
with the infiltration of inflammatory cells. It was shown in vitro that MMP-2 and MMP-9 activate interleukin (IL)-1 $\beta$ by its cleaving the precursor pro-IL- $1 \beta .{ }^{68}$ Then, IL- $1 \beta$ stimulates proliferation of VSMC and the formation of reactive oxygen species in arteries, thus contributing to generate hypertension. ${ }^{69}$ Figure 1 illustrates MMP-2 cleaving IL-1 $\beta$ in VSMC. Increased MMP activity also contributes to reduce the levels of P-selectin in the endothelial cells of SHR rats (Figure 1). $\mathrm{P}$-selectin is a transient adhesion receptor that mediates leukocyte adhesion during inflammatory conditions. Treating SHR with an MMP inhibitor attenuated the loss of P-selectin in post-capillary endothelium of mesenteric arteries and improved the immune response in hypertension. ${ }^{68} \mathrm{MMP}-2$ also degrades the monocyte chemoattractant protein-3 and thereby generates a chemokine receptor antagonist that decreases leukocyte migration. ${ }^{69}$ Incubation of MMP-9 and MMP-7 with inferior vena cava and internal jugular veins of SHR rats showed that both proteases cleave the extracellular portion of intracellular adhesion molecule (ICAM-1). As ICAM-1 facilitates leukocyte infiltration and increased production of inflammatory cytokines, its proteolytic cleavage is important to reduce some inflammatory stages of hypertension. ${ }^{72}$

\section{Intracellular targets of MMP-2 in the cardiovascular system}

MMP-2 is potentially activated intracellularly by oxidative stress and contributes to the proteolysis of structural and contractile proteins into the cardiomyocytes and VSMC that leads to cardiovascular dysfunction in many diseases. The intracellular role of MMP-2 was first observed in isolated rat hearts submitted to ischemia and reperfusion injury, in which MMP-2 degraded troponin I in the sarcomere of cardiomyocytes. ${ }^{10}$ It was also shown that troponin I is colocalized with MMP-2 in the thin filaments of cardiomyocytes and is cleaved by MMP-2 in vitro. Treatment with MMP inhibitors restored the cardiac levels of troponin I and improved the recovery of contractile function post-ischemia and reperfusion. ${ }^{12}$ Also, increased MMP-2 activity proteolyzes cardiac $\alpha$-actinin, ${ }^{73}$ myosin light chain- $1^{74}$ and titin, ${ }^{11}$ the molecular spring of sarcomere, during ischemia and reperfusion injury. ${ }^{75}$ These effects were prevented by using MMP inhibitors or by genetic ablation of MMP-2. Dystrophin is a cytoskeleton protein that connects the ECM to the sarcomere in cardiomyocytes. It was observed that increased MMP-2 activity was associated with loss of dystrophin and cardiac dysfunction in rabbit hearts subjected to ischemia and reperfusion injury. In fact, treatment with doxycycline improved the cardiac function by decreasing MMP-2 activity and dystrophin breakdown. ${ }^{76}$ In a study of patients with coronary artery disease undergoing coronary bypass graft surgery, treatment with a subantimicrobial dose of doxycycline notably reduced increased cardiac MMP-2 activity, although it did not improve myocardial stunning following this condition. ${ }^{77}$

MMP-2 is also activated in the vasculature during oxidative stress conditions and contributes to the proteolysis of intracellular proteins. Calponin-1 was the first target of MMP-2 analyzed in vitro and in the vasculature of endotoxemic and hypertensive rats. ${ }^{1}$ Calponin- 1 is an actin-binding protein located in the contractile machinery of VSMC and contains regions of sequence homology to cardiac troponins. ${ }^{76,77}$ Figure 1 shows an intracellular form of MMP-2 into VSMC and its proteolytic role on calponin-1 in the contractile machinery. In rats treated with LPS, increased MMP-2 activity decreased protein levels of calponin-1 in aortas, which contributed to a significant hypocontractility. When LPS rats were treated with doxycycline, the levels of calponin-1 were restored as well as aorta contractility. Furthermore, as calponin-1 is also a cytoskeleton protein and a differentiation marker of VSMC, its loss further contributes to a phenotype switch of VSMC from contractile to synthetic, thus leading to cell migration and proliferation. ${ }^{80,81}$ In fact, we recently showed that increased activity of MMP-2 in aortas of 1 -week-old $2 \mathrm{~K}-1 \mathrm{C}$ rats was associated with reduced expression of protein levels of calponin-1 and increased VSMC proliferation, which then preceded hypertensioninduced arterial hypertrophic remodeling. ${ }^{1,2}$ When $2 \mathrm{~K}-1 \mathrm{C}$ rats were treated with doxycycline, both calponin-1 loss and proliferation of VSMC were prevented. As we did not observe any alterations in myocardin protein levels and calponin-1 mRNA in aortas of hypertensive rats, the loss of calponin-1 may be attributed to its proteolysis by MMP-2. ${ }^{1}$ In a model of porcine aorta coarctation, reduction in the protein levels of calponin, smoothelin and caldesmon were associated with increased proliferation of VSMC and the development of hypertension. ${ }^{82}$ Moreover, deoxycorticosterone acetatesalt rats also showed increased proliferation of VSMC and decreased calponin-1 and myocardin levels in aortas. ${ }^{83}$ Since MMP-2 may be intracellularly activated and causes dysfunction in VSMC and cardiomyocytes, pharmacological tools that precisely inhibit its intracellular forms could be attractive approaches to avoid cardiovascular dysfunction and chronic remodeling in hypertension. More studies are warranted to investigate this hypothesis. 


\section{Antioxidants and antihypertensive drugs ameliorate hypertension- induced chronic remodeling and dysfunction by decreasing MMP activity}

As hypertension is associated with oxidative stress and MMPs can be activated by reactive oxygen species to produce chronic vascular remodeling and dysfunction, the use of antioxidants may control hypertension by inhibiting MMPs. In fact, treatment of $2 \mathrm{~K}-1 \mathrm{C}$ rats with tempol reduced systolic blood pressure, oxidative stress and increased MMP-2 activity in aortas, thus ameliorating endothelium dysfunction and chronic vascular remodeling. ${ }^{4}$ Tempol also decreased cardiac hypertrophy in $2 \mathrm{~K}-1 \mathrm{C}$ rats by decreasing TGF- $\beta$ levels, oxidative stress and MMP-2 activity. ${ }^{53}$ Some natural antioxidants are also effective in inhibiting MMP activity. Red wine polyphenols prevented an increase in systolic blood pressure in rats caused by angiotensin II by reducing aortic oxidative stress and MMP-2 activity. ${ }^{84}$ Furthermore, by using perinatal maternal food supplementation with resveratrol, the development of hypertension in newborn SHR was attenuated by its antioxidant effect. ${ }^{85}$ Quercetin, a polyphenol that is present in green vegetables and fruits, decreased MMP-2 activity by reducing oxidative stress in a model of mouse abdominal aortic aneurysm ${ }^{86}$ and recovered cardiac function after ischemia in hearts of doxorubicin-treated rats. ${ }^{87}$ Quercetin also improved hypertension-induced vascular remodeling by decreasing oxidative stress and MMP-2 activity in aortas of $2 \mathrm{~K}-1 \mathrm{C}$ rats. ${ }^{88}$ A vegetarian diet was also associated with reduced plasma levels of MMP-2 and MMP-9 in healthy human patients, thus suggesting that a diet rich in flavonols may decrease the risk of developing cardiovascular diseases by reducing MMP. ${ }^{89}$ Doxycycline also had antioxidant properties when used in $2 \mathrm{~K}-1 \mathrm{C}$ rats, and this effect may improve its capacity to reduce MMP activity and expression. Doxycycline ameliorated aortic endothelial dysfunction, decreased oxidative stress and MMP-2 activity, and increased nitric oxide bioavailability in $2 \mathrm{~K}-1 \mathrm{C}$ rats. ${ }^{90}$ Figure 1 also shows the antioxidants reducing the capacity of oxidative stress to potentially activate the intracellular MMP-2 and its effects on calponin-1 in the VSMC.

Activation of the renin-angiotensin system is involved in the genesis of hypertension and pharmacological therapies that target this system are nowadays widely used to treat hypertension. However, part of the antihypertensive effects of these drugs is due to the reduction of oxidative stress and MMP activity. In fact, the losartan metabolite inhibited NADPH oxidase activity when incubated in vitro with human phagocytic cells, and then ameliorated the oxidative stress scenario and decreased MMP-9 secretion from these cells. Decreased levels of MMP-9 are also observed in plasma of hypertensive patients who are treated with losartan. ${ }^{91} \mathrm{MMP}-2$ activity is differently modulated by angiotensin II and depends whether angiotensin II binds to $\mathrm{AT}_{1}$ or $\mathrm{AT}_{2}$ receptors. Blockade of $\mathrm{AT}_{1}$ with losartan improved VSMC growth and the stiffness of mesenteric arteries in angiotensin II infused rats; however, the concomitant blockade of $\mathrm{AT}_{1}$ and $\mathrm{AT}_{2}$ receptors showed an increase in remodeling and increased MMP-2 activity in mesenteric arteries. Therefore, the AT receptor activation seems to be involved in the increased vascular stiffness of hypertension while $\mathrm{AT}_{2}$ receptor activation has a protective effect and stimulates a reduction in MMP-2 activity. ${ }^{92}$ Furthermore, blockade of angiotensin receptor $\mathrm{AT}_{1}$ seems to be more effective in preventing arterial maladaptive morphological changes in hypertension than the direct inhibition of renin or angiotensin converting enzyme..$^{93}$ Treatment with losartan or in combination with aliskiren in $2 \mathrm{~K}-1 \mathrm{C}$ rats ameliorated hypertension-induced aortic stiffness by reducing the deposition of collagen. These treatments reduced the increased MMP- 2 activity, the expression of phospho-ERK $1 / 2$ and the levels of TGF- $\beta$ in aortas of hypertensive rats. These effects were not observed when rats were treated with aliskiren alone. ${ }^{93}$ On the other hand, enalapril did not reduce plasma levels of MMP-2, MMP-8, MMP-9, TIMP-1 and TIMP-2, or MMP activity in human hypertensive patients..$^{94}$ Moreover, losartan, but not ramipril, decreased MMP-2 levels in plasma of hypertensive patients. ${ }^{95}$

The calcium channel blockers also showed inhibitory effects on MMP activity in hypertension by attenuating oxidative stress (Figure 1). Treatment with subhypertensive or antihypertensive doses of nifedipine decreased cardiac oxidative stress, MMP-2 activity and the ratio of collagen type I to type III in Dahl salt-sensitive rats, in addition to reduce left ventricular fibrosis and diastolic heart failure. ${ }^{96}$ Treatment with lercanidipine in the $2 \mathrm{~K}-1 \mathrm{C}$ rats ameliorated aortic endothelial dysfunction, oxidative stress and the increased MMP-2 activity. ${ }^{97}$ Furthermore, treatment with nifedipine, nimodipine and amlodipine prevented $2 \mathrm{~K}-1 \mathrm{C}$ rat-induced aortic hypertrophy by decreasing oxidative stress and MMP-2 activity. ${ }^{98}$

$\beta$-Blockers and diuretics are also efficient in ameliorating hypertension-induced cardiac and vascular morphological changes by reducing oxidative stress and MMP activity (Figure 1). Treatment with nebivolol and metoprolol attenuated the increased MMP-2 activity, oxidative stress, collagen deposition and cardiac hypertrophy in $2 \mathrm{~K}-1 \mathrm{C}$ rats. ${ }^{99}$ 
However, only nebivolol improved aortic vascular remodeling in these rats by decreasing oxidative stress, MMP-2 activity and the increased levels of TGF- $\beta .{ }^{55}$ Spironolactone and hydrochlorothiazide exerted antioxidant effects and ameliorated aortic vascular remodeling and dysfunction by reducing increased MMP-2 activity in $2 \mathrm{~K}-1 \mathrm{C}$ rats. ${ }^{100}$ Moreover, spironolactone improved cardiac hypertrophy and dysfunction after infusion of isoproterenol in SHR rats. These effects were associated with decreased MMP-2 activity and cardiac collagen deposition, which prevented the transition of left ventricle hypertrophic remodeling to dilation and heart failure. ${ }^{101}$

\section{Conclusion}

Increased MMP activity, primarily that of MMP-2, contributes to hypertension-induced vascular and cardiac maladaptive alterations by degrading ECM, non-ECM and inflammatory components in both VSMC and cardiomyocytes. The intracellular effects of MMP-2 also contribute to cause cell hypertrophy and/or dysfunction, which result in the maladaptive consequences of hypertension. Direct inhibition of MMP-2 activity and indirect inhibition by reducing oxidative stress are important strategies that may improve hypertension and its cardiovascular complications. New pharmacological tools are needed to specifically target the intracellular forms of MMP-2 and then contribute to reduce cell migration and/or hypertrophy in hypertension and many other cardiovascular diseases.

\section{Acknowledgments}

Our studies are funded by Fundação de Amparo a Pesquisa do Estado de São Paulo (FAPESP), Conselho Nacional de Desenvolvimento Científico e Tecnológico (CNPQ) and Coordenação de Aperfeiçoamento de Pessoal de Nível Superior (CAPES). JMP is a PhD student of CAPES. We kindly thank Marcelo H. Milan for graphical assistance and Prof. Dr Richard Schulz (University of Alberta, Edmonton, Canada) for his helpful comments in the manuscript.

\section{Disclosure}

The authors report no conflicts of interest in this work.

\section{References}

1. Belo VA, Parente JM, Tanus-Santos JE, Castro MM. Matrix metalloproteinase (MMP)-2 decreases calponin-1 levels and contributes to arterial remodeling in early hypertension. Biochem Pharmacol. 2016;118:50-58.

2. Parente JM, Pereira CA, Oliveira-Paula GH, Tanus-Santos JE, Tostes $\mathrm{RC}$, Castro MM. Matrix metalloproteinase-2 activity is associated with divergent regulation of calponin-1 in conductance and resistance arteries in hypertension-induced early vascular dysfunction and remodelling. Basic Clin Pharmacol Toxicol. 2017;121:246-256.
3. Castro MM, Rizzi E, Figueiredo-Lopes L, et al. Metalloproteinase inhibition ameliorates hypertension and prevents vascular dysfunction and remodeling in renovascular hypertensive rats. Atherosclerosis. 2008;198:320-331.

4. Castro MM, Rizzi E, Rodrigues GJ, et al. Antioxidant treatment reduces matrix metalloproteinase-2-induced vascular changes in renovascular hypertension. Free Radic Biol Med. 2009;46:1298-1307.

5. Castro MM, Rizzi E, Prado CM, Rossi MA, Tanus-Santos JE, Gerlach RF. Imbalance between matrix metalloproteinases and tissue inhibitor of metalloproteinases in hypertensive vascular remodeling. Matrix Biol. 2010;29:194-201.

6. Ceron CS, Rizzi E, Guimaraes DA, et al. Time course involvement of matrix metalloproteinases in the vascular alterations of renovascular hypertension. Matrix Biol. 2012;31:261-270.

7. Guimaraes DA, Rizzi E, Ceron CS, et al. Doxycycline dose-dependently inhibits MMP-2-mediated vascular changes in 2K1C hypertension. Basic Clin Pharmacol Toxicol. 2011;108:318-325.

8. Rizzi E, Castro MM, Prado CM, et al. Matrix metalloproteinase inhibition improves cardiac dysfunction and remodeling in 2-kidney, 1-clip hypertension. J Card Fail. 2010;16:599-608.

9. Chen Q, Jin M, Yang F, Zhu J, Xiao Q, Zhang L. Matrix metalloproteinases: inflammatory regulators of cell behaviors in vascular formation and remodeling. Mediators Inflamm. 2013;2013:928315.

10. Schulz R. Intracellular targets of matrix metalloproteinase- 2 in cardiac disease: rationale and therapeutic approaches. Annu Rev Pharmacol Toxicol. 2007;47:211-242.

11. Ali MA, Cho WJ, Hudson B, Kassiri Z, Granzier H, Schulz R. Titin is a target of matrix metalloproteinase-2: implications in myocardial ischemia/reperfusion injury. Circulation. 2010;122:2039-2047.

12. Wang W, Schulze CJ, Suarez-Pinzon WL, Dyck JR, Sawicki G, Schulz R. Intracellular action of matrix metalloproteinase-2 accounts for acute myocardial ischemia and reperfusion injury. Circulation. 2002;106:1543-1549.

13. Gross J, Lapiere CM. Collagenolytic activity in amphibian tissues: a tissue culture assay. Proc Natl Acad Sci U S A. 1962;48: 1014-1022.

14. Page-McCaw A, Ewald AJ, Werb Z. Matrix metalloproteinases and the regulation of tissue remodelling. Nat Rev Mol Cell Biol. 2007;8:221-233.

15. Galis ZS, Khatri JJ. Matrix metalloproteinases in vascular remodeling and atherogenesis: the good, the bad, and the ugly. Circ Res. 2002;90:251-262.

16. Visse R, Nagase H. Matrix metalloproteinases and tissue inhibitors of metalloproteinases: structure, function, and biochemistry. Circ Res. 2003;92:827-839.

17. Nagase H, Visse R, Murphy G. Structure and function of matrix metalloproteinases and TIMPs. Cardiovasc Res. 2006;69:562-573.

18. Amin M, Pushpakumar S, Muradashvili N, Kundu S, Tyagi SC, Sen U. Regulation and involvement of matrix metalloproteinases in vascular diseases. Front Biosci (Landmark Ed). 2016;21:89-118.

19. Overall CM. Molecular determinants of metalloproteinase substrate specificity: matrix metalloproteinase substrate binding domains, modules, and exosites. Mol Biotechnol. 2002;22:51-86.

20. Nagase H, Woessner JF Jr. Matrix metalloproteinases. J Biol Chem. 1999;274:21491-21494.

21. Ra HJ, Parks WC. Control of matrix metalloproteinase catalytic activity. Matrix Biol. 2007;26:587-596.

22. Belo VA, Guimarães DA, Castro MM. Matrix metalloproteinase 2 as a potential mediator of vascular smooth muscle cell migration and chronic vascular remodeling in hypertension. J Vasc Res. 2015;52:221-231.

23. Sternlicht MD, Werb Z. How matrix metalloproteinases regulate cell behavior. Annu Rev Cell Dev Biol. 2001;17:463-516.

24. Lovett DH, Mahimkar R, Raffai RL, et al. N-terminal truncated intracellular matrix metalloproteinase- 2 induces cardiomyocyte hypertrophy, inflammation and systolic heart failure. PLoS One. 2013;8:e68154. 
25. Ali MA, Chow AK, Kandasamy AD, et al. Mechanisms of cytosolic targeting of matrix metalloproteinase-2. J Cell Physiol. 2012;227: 3397-3404.

26. Viappiani S, Nicolescu AC, Holt A, et al. Activation and modulation of $72 \mathrm{kDa}$ matrix metalloproteinase- 2 by peroxynitrite and glutathione. Biochem Pharmacol. 2009;77:826-834.

27. Sariahmetoglu M, Crawford BD, Leon H, et al. Regulation of matrix metalloproteinase-2 (MMP-2) activity by phosphorylation. FASEB J. 2007;21:2486-2495.

28. Jacob-Ferreira AL, Kondo MY, Baral PK, et al. Phosphorylation status of $72 \mathrm{kDa}$ MMP-2 determines its structure and activity in response to peroxynitrite. PLoS One. 2013;8:e71794

29. Brew K, Dinakarpandian D, Nagase H, Tissue inhibitors of metalloproteinases: evolution, structure and function. Biochim Biophys Acta. 2000;1477:267-283

30. Schulze CJ, Wang W, Suarez-Pinzon WL, Sawicka J, Sawicki G, Schulz R. Imbalance between tissue inhibitor of metalloproteinase-4 and matrix metalloproteinases during acute myocardial [correction of myoctardial] ischemia-reperfusion injury. Circulation. 2003; 107:2487-2492.

31. Golub LM, Lee HM, Lehrer G, et al. Minocycline reduces gingival collagenolytic activity during diabetes. Preliminary observations and a proposed new mechanism of action. J Periodontal Res. 1983; 18:516-526.

32. Golub LM, Goodson JM, Lee HM, Vidal AM, McNamara TF, Ramamurthy NS. Tetracyclines inhibit tissue collagenases. Effects of ingested low-dose and local delivery systems. J Periodontol. 1985;56:93-97.

33. Golub LM, McNamara TF, D’Angelo G, Greenwald RA, Ramamurthy NS. A non-antibacterial chemically-modified tetracycline inhibits mammalian collagenase activity. J Dent Res. 1987;66: 1310-1314.

34. Hu J, Van den Steen PE, Sang QX, Opdenakker G. Matrix metalloproteinase inhibitors as therapy for inflammatory and vascular diseases. Nat Rev Drug Discov. 2007;6:480-498.

35. Rossello A, Nuti E, Orlandini E, et al. New N-arylsulfonyl-N-alkoxyaminoacetohydroxamic acids as selective inhibitors of gelatinase A (MMP-2). Bioorg Med Chem. 2004;12:2441-2450.

36. Yamada A, Uegaki A, Nakamura T, Ogawa K. ONO-4817, an orally active matrix metalloproteinase inhibitor, prevents lipopolysaccharideinduced proteoglycan release from the joint cartilage in guinea pigs. Inflamm Res. 2000;49:144-146.

37. Gatto C, Rieppi M, Borsotti P, et al. BAY 12-9566, a novel inhibitor of matrix metalloproteinases with antiangiogenic activity. Clin Cancer Res. 1999;5:3603-3607.

38. Golub LM, Sorsa T, Lee HM, et al. Doxycycline inhibits neutrophil (PMN)-type matrix metalloproteinases in human adult periodontitis gingiva. J Clin Periodontol. 1995;22:100-109.

39. Castro MM, Tanus-Santos JE. Inhibition of matrix metalloproteinases (MMPs) as a potential strategy to ameliorate hypertensioninduced cardiovascular alterations. Curr Drug Targets. 2013;14: 335-343.

40. Hopps E, Caimi G. Matrix metalloproteases as a pharmacological target in cardiovascular diseases. Eur Rev Med Pharmacol Sci. 2015;19:2583-2589.

41. Hughes BG, Schulz R. Targeting MMP-2 to treat ischemic heart injury. Basic Res Cardiol. 2014;109:424.

42. Martinez-Lemus LA, Hill MA, Meininger GA. The plastic nature of the vascular wall: a continuum of remodeling events contributing to control of arteriolar diameter and structure. Physiology (Bethesda). 2009;24:45-57.

43. Mulvany MJ. Vascular remodelling of resistance vessels: can we define this? Cardiovasc Res. 1999;41:9-13.

44. Briones AM, Xavier FE, Arribas SM, et al. Alterations in structure and mechanics of resistance arteries from ouabain-induced hypertensive rats. Am J Physiol Heart Circ Physiol. 2006;291:H193-H201.
45. Bouvet C, Gilbert LA, Girardot D, deBlois D, Moreau P. Different involvement of extracellular matrix components in small and large arteries during chronic NO synthase inhibition. Hypertension. 2005;45:432-437.

46. Wang M, Zhang J, Telljohann R, et al. Chronic matrix metalloproteinase inhibition retards age-associated arterial proinflammation and increase in blood pressure. Hypertension. 2012;60:459-466.

47. Chesler NC, Ku DN, Galis ZS. Transmural pressure induces matrix-degrading activity in porcine arteries ex vivo. Am J Physiol. 1999;277:H2002-H2009.

48. Gjesdal O, Bluemke DA, Lima JA. Cardiac remodeling at the population level--risk factors, screening, and outcomes. Nat Rev Cardiol. 2011;8:673-685.

49. Liu P, Sun M, Sader S. Matrix metalloproteinases in cardiovascular disease. Can J Cardiol. 2006;22(Suppl B):25B-30B.

50. Wang M, Kim SH, Monticone RE, Lakatta EG. Matrix metalloproteinases promote arterial remodeling in aging, hypertension, and atherosclerosis. Hypertension. 2015;65:698-703.

51. Bergman MR, Teerlink JR, Mahimkar R, et al. Cardiac matrix metalloproteinase-2 expression independently induces marked ventricular remodeling and systolic dysfunction. Am J Physiol Heart Circ Physiol. 2007;292:H1847-H1860.

52. Ruiz-Ortega M, Rodriguez-Vita J, Sanchez-Lopez E, Carvajal G, Egido J. TGF-beta signaling in vascular fibrosis. Cardiovasc Res. 2007;74:196-206.

53. Rizzi E, Castro MM, Ceron CS, et al. Tempol inhibits TGF- $\beta$ and MMPs upregulation and prevents cardiac hypertensive changes. Int $J$ Cardiol. 2013;165:165-173.

54. Wang M, Zhao D, Spinetti G, et al. Matrix metalloproteinase 2 activation of transforming growth factor-beta1 (TGF-beta1) and TGF-beta1type II receptor signaling within the aged arterial wall. Arterioscler Thromb Vasc Biol. 2006;26:1503-1509.

55. Ceron CS, Rizzi E, Guimarães DA, Martins-Oliveira A, Gerlach RF, Tanus-Santos JE. Nebivolol attenuates prooxidant and profibrotic mechanisms involving TGF- $\beta$ and MMPs, and decreases vascular remodeling in renovascular hypertension. Free Radic Biol Med. 2013;65:47-56

56. Iwazu Y, Muto S, Hirahara I, Fujisawa G, Takeda S, Kusano E. Matrix metalloproteinase 2 induces epithelial-mesenchymal transition in proximal tubules from the luminal side and progresses fibrosis in mineralocorticoid/salt-induced hypertensive rats. J Hypertens. 2011;29:2440-2453.

57. Dwivedi A, Slater SC, George SJ. MMP-9 and -12 cause N-cadherin shedding and thereby $\beta$-catenin signalling and vascular smooth muscle cell proliferation. Cardiovasc Res. 2009;81:178-186.

58. Fernandez-Patron C, Radomski MW, Davidge ST. Vascular matrix metalloproteinase-2 cleaves big endothelin-1 yielding a novel vasoconstrictor. Circ Res. 1999;85:906-911.

59. Abdalvand A, Morton JS, Bourque SL, Quon AL, Davidge ST. Matrix metalloproteinase enhances big-endothelin-1 constriction in mesenteric vessels of pregnant rats with reduced uterine blood flow. Hypertension. 2013;61:488-493.

60. Fernandez-Patron C, Stewart KG, Zhang Y, Koivunen E, Radomski MW, Davidge ST. Vascular matrix metalloproteinase-2-dependent cleavage of calcitonin gene-related peptide promotes vasoconstriction. Circ Res. 2000;87:670-676.

61. Martínez A, Oh HR, Unsworth EJ, et al. Matrix metalloproteinase-2 cleavage of adrenomedullin produces a vasoconstrictor out of a vasodilator. Biochem J. 2004;383:413-418.

62. Nascimento RA, Mendes G, Possomato-Vieira JS, et al. Metalloproteinase inhibition protects against reductions in circulating adrenomedullin during lead-induced acute hypertension. Basic Clin Pharmacol Toxicol. 2015;116:508-515.

63. Nagareddy PR, Rajput PS, Vasudevan H, et al. Inhibition of matrix metalloproteinase-2 improves endothelial function and prevents hypertension in insulin-resistant rats. Br J Pharmacol. 2012;165:705-715. 
64. Rodrigues SF, Tran ED, Fortes ZB, Schmid-Schönbein GW. Matrix metalloproteinases cleave the beta2-adrenergic receptor in spontaneously hypertensive rats. Am J Physiol Heart Circ Physiol. 2010;299: H25-H35.

65. DeLano FA, Schmid-Schönbein GW. Proteinase activity and receptor cleavage: mechanism for insulin resistance in the spontaneously hypertensive rat. Hypertension. 2008;52:415-423.

66. Tran ED, DeLano FA, Schmid-Schönbein GW. Enhanced matrix metalloproteinase activity in the spontaneously hypertensive rat: VEGFR-2 cleavage, endothelial apoptosis, and capillary rarefaction. JVasc Res. 2010;47:423-431.

67. Barhoumi T, Fraulob-Aquino J, Mian MOR, et al. Matrix metalloproteinase-2 knockout prevents angiotensin II-induced vascular injury. Cardiovasc Res. 2017;113:1753-1762.

68. Schönbeck U, Mach F, Libby P. Generation of biologically active IL-1 beta by matrix metalloproteinases: a novel caspase-1-independent pathway of IL-1 beta processing. J Immunol. 1998;161:3340-3346.

69. Krishnan SM, Sobey CG, Latz E, Mansell A, Drummond GR. IL-1 $\beta$ and IL-18: inflammatory markers or mediators of hypertension? Br J Pharmacol. 2014;171:5589-5602.

70. Chen AY, Ha JN, Delano FA, Schmid-Schönbein GW. Receptor cleavage and P-selectin-dependent reduction of leukocyte adhesion in the spontaneously hypertensive rat. J Leukoc Biol. 2012;92:183-194.

71. McQuibban GA, Gong JH, Tam EM, McCulloch CA, Clark-Lewis I, Overall CM. Inflammation dampened by gelatinase A cleavage of monocyte chemoattractant protein-3. Science. 2000;289:1202-1206.

72. Tong S, Neboori HJ, Tran ED, Schmid-Schönbein GW. Constitutive expression and enzymatic cleavage of ICAM-1 in the spontaneously hypertensive rat. J Vasc Res. 2011;48:386-396.

73. Sung MM, Schulz CG, Wang W, Sawicki G, Bautista-López NL, Schulz R. Matrix metalloproteinase-2 degrades the cytoskeletal protein alpha-actinin in peroxynitrite mediated myocardial injury. $\mathrm{J} \mathrm{Mol} \mathrm{Cell}$ Cardiol. 2007;43:429-436.

74. Sawicki G, Leon H, Sawicka J, et al. Degradation of myosin light chain in isolated rat hearts subjected to ischemia-reperfusion injury: a new intracellular target for matrix metalloproteinase-2. Circulation. 2005;112:544-552.

75. Ali MA, Fan X, Schulz R. Cardiac sarcomeric proteins: novel intracellular targets of matrix metalloproteinase-2 in heart disease. Trends Cardiovasc Med. 2011;21:112-118.

76. Buchholz B, Perez V, Siachoque N, et al. Dystrophin proteolysis: a potential target for MMP-2 and its prevention by ischemic preconditioning. Am J Physiol Heart Circ Physiol. 2014;307:H88-H96.

77. Schulze CJ, Castro MM, Kandasamy AD, et al. Doxycycline reduces cardiac matrix metalloproteinase-2 activity but does not ameliorate myocardial dysfunction during reperfusion in coronary artery bypass patients undergoing cardiopulmonary bypass. Crit Care Med. 2013;41:2512-2520.

78. Takahashi K, Hiwada K, Kokubu T. Vascular smooth muscle calponin. A novel troponin T-like protein. Hypertension. 1988;11:620-626.

79. Winder SJ, Walsh MP. Smooth muscle calponin. Inhibition of actomyosin MgATPase and regulation by phosphorylation. J Biol Chem. 1990;265:10148-10155.

80. North AJ, Gimona M, Cross RA, Small JV. Calponin is localised in both the contractile apparatus and the cytoskeleton of smooth muscle cells. J Cell Sci. 1994;107(Pt 3):437-444.

81. Gimona M, Herzog M, Vandekerckhove J, Small JV. Smooth muscle specific expression of calponin. FEBS Lett. 1990;274:159-162.

82. Hu JJ, Ambrus A, Fossum TW, Miller MW, Humphrey JD, Wilson E. Time courses of growth and remodeling of porcine aortic media during hypertension: a quantitative immunohistochemical examination. J Histochem Cytochem. 2008;56:359-370.

83. Pfisterer L, Feldner A, Hecker M, Korff T. Hypertension impairs myocardin function: a novel mechanism facilitating arterial remodelling. Cardiovasc Res. 2012;96:120-129.
84. Walter A, Etienne-Selloum N, Sarr M, Kane MO, Beretz A, SchiniKerth VB. Angiotensin II induces the vascular expression of VEGF and MMP-2 in vivo: preventive effect of red wine polyphenols. JVasc Res. 2008;45:386-394.

85. Care AS, Sung MM, Panahi S, et al. Perinatal resveratrol supplementation to spontaneously hypertensive rat dams mitigates the development of hypertension in adult offspring. Hypertension. 2016;67:1038-1044.

86. Wang L, Cheng X, Li H, et al. Quercetin reduces oxidative stress and inhibits activation of cJun N-terminal kinase/activator protein-1 signaling in an experimental mouse model of abdominal aortic aneurysm. Mol Med Rep. 2014;9:435-442.

87. Barteková M, Šimončíková P, Fogarassyová M, et al. Quercetin improves postischemic recovery of heart function in doxorubicintreated rats and prevents doxorubicin-induced matrix metalloproteinase-2 activation and apoptosis induction. Int J Mol Sci. 2015; 16:8168-8185.

88. Pereira, SC, Parente, JM, Belo, VA, et al., Quercetin decreases the activity of matrix metalloproteinase-2 and ameliorates vascular remodeling in renovascular hypertension. Atherosclerosis. 2018;270: $146-153$.

89. Navarro JA, de Gouveia LA, Rocha-Penha L, et al. Reduced levels of potential circulating biomarkers of cardiovascular diseases in apparently healthy vegetarian men. Clin Chim Acta. 2016;461:110-113.

90. Castro MM, Rizzi E, Ceron CS, et al. Doxycycline ameliorates $2 \mathrm{~K}-1 \mathrm{C}$ hypertension-induced vascular dysfunction in rats by attenuating oxidative stress and improving nitric oxide bioavailability. Nitric Oxide. 2012;26:162-168.

91. Fortuño A, Bidegain J, Robador PA, et al. Losartan metabolite EXP3179 blocks NADPH oxidase-mediated superoxide production by inhibiting protein kinase $\mathrm{C}$ : potential clinical implications in hypertension. Hypertension. 2009;54:744-750.

92. Brassard P, Amiri F, Schiffrin EL. Combined angiotensin II type 1 and type 2 receptor blockade on vascular remodeling and matrix metalloproteinases in resistance arteries. Hypertension. 2005;46:598-606.

93. Martins-Oliveira A, Castro MM, Oliveira DM, et al. Contrasting effects of aliskiren versus losartan on hypertensive vascular remodeling. Int J Cardiol. 2013;167:1199-1205.

94. Fontana V, Silva PS, Izidoro-Toledo TC, et al. Comprehensive evaluation of the effects of enalapril on matrix metalloproteinases levels in hypertension. Cardiovasc Drugs Ther. 2012;26:511-519.

95. Derosa G, Maffioli P, Ferrari I, et al. Different actions of losartan and ramipril on adipose tissue activity and vascular remodeling biomarkers in hypertensive patients. Hypertens Res. 2011;34:145-151.

96. Yamada T, Nagata K, Cheng XW, et al. Long-term administration of nifedipine attenuates cardiac remodeling and diastolic heart failure in hypertensive rats. Eur J Pharmacol. 2009;615:163-170.

97. Martinez ML, Castro MM, Rizzi E, et al. Lercanidipine reduces matrix metalloproteinase-2 activity and reverses vascular dysfunction in renovascular hypertensive rats. Eur J Pharmacol. 2008;591:224-230.

98. Marçal DM, Rizzi E, Martins-Oliveira A, et al. Comparative study on antioxidant effects and vascular matrix metalloproteinase-2 downregulation by dihydropyridines in renovascular hypertension. Naunyn Schmiedebergs Arch Pharmacol. 2011;383:35-44.

99. Rizzi E, Guimaraes DA, Ceron CS, et al. $\beta 1$-Adrenergic blockers exert antioxidant effects, reduce matrix metalloproteinase activity, and improve renovascular hypertension-induced cardiac hypertrophy. Free Radic Biol Med. 2014;73:308-317.

100. Ceron CS, Castro MM, Rizzi E, et al. Spironolactone and hydrochlorothiazide exert antioxidant effects and reduce vascular matrix metalloproteinase-2 activity and expression in a model of renovascular hypertension. Br J Pharmacol. 2010;160:77-87.

101. Veliotes DG, Norton GR, Correia RJ, et al. Impact of aldosterone receptor blockade on the deleterious cardiac effects of adrenergic activation in hypertensive rats. $J$ Cardiovasc Pharmacol. 2010;56: 203-211. 
Metalloproteinases In Medicine is an international, peer reviewed, open access journal that aims to provide a platform for the discussion and dissemination of knowledge about the role that metalloproteinases - such as matrix metalloproteinases (MMP), ADAMs, ADAMTSs, and astacins, as well as their inhibitors - play in diseases.

\section{Dovepress}

The manuscript management system is completely online and includes a very quick and fair peer review system, which is all easy to use. Visit http://www.dovepress.com/testimonials.php to read real quotes from published authors.

Submit your manuscript here: https://www.dovepress.com/metalloproteinases-in-medicine-journal 